\title{
Construction of the ground state of Matrix Theory: Near the Origin
}

\section{Maciej TRZETRZELEWSKI ${ }^{*}$}

Jagiellonian University

E-mail: 33 lewski@th.if.uj.edu.pl

A unique $\operatorname{Spin}(9) \times S U(2)$ singlet state, involving only the fermionic degrees of freedom, corresponding to the supermembrane/M-theory matrix-model, is constructed. Any ground state of the theory evaluated at the origin must be proportional to the state.

European Physical Society Europhysics Conference on High Energy Physics

July 16-22, 2009

Krakow, Poland

${ }^{*}$ Speaker. 
In early $60^{\prime}$ Dirac considered a possibility that the electron could be described by a conducting membrane with a spherical topology [1]. His motivation was to explain the existence of the muon and its mass in terms of the excitation of the ground state identified with the electron. Ironically, although Dirac's findings did not match the experiential data, the modifications of the membraneaction he considered are nowadays one of the key tools in analyzing the D-brane dynamics.

As found by Goldstone and Hoppe [2] the quantum theory of the Dirac's membrane can be formulated by takeing the large $N$ limit of a certain matrix model of $N \times N$ matrices. The supersymmetric version of that model [3] has an intriguing property: not only does it describe the supermembrane but also it is nicely related to M-theory by the BFSS conjecture [4]. This relation makes the supermembrane matrix theory very attractive also in string theory.

The supermembrane matrix theory is a quantum mechanical model with $\mathscr{N}=16$ supersymmetries, $S U(N)$ gauge invariance and $\operatorname{Spin}(9)$ symmetry. The theory involves real bosonic variables $x_{s A}$ (coordinates) and real fermionic ones $\theta_{\alpha A}$ (Majorana spinors) with $s=1, \ldots, 9, \alpha=1, \ldots 16$ and $A=1, \ldots, N^{2}-1$ - spatial, spinor and color indices respectively. The corresponding supercharges and the Hamiltonian of the model are

$$
\begin{gathered}
\mathscr{Q}_{\alpha}=\left(-i \partial_{s A} \gamma_{\alpha \beta}^{s}+\frac{1}{2} f_{A B C} x_{s B} x_{t C} \gamma_{\alpha \beta}^{s t}\right) \theta_{\beta A}, \quad \gamma^{s t}=\frac{1}{2}\left[\gamma^{s}, \gamma^{t}\right], \\
H=-\partial_{s A} \partial_{s A}+\frac{1}{2}\left(f_{A B C} x_{s B} x_{t C}\right)^{2}+i f_{A B C} \gamma_{\alpha \beta}^{s} \theta_{\alpha A} \theta_{\beta B} x_{s C}, \\
\left\{\mathscr{Q}_{\alpha}, \mathscr{Q}_{\beta}\right\}=\delta_{\alpha \beta} H+2 \gamma_{\alpha \beta}^{s} x_{s A} J_{A}, \quad J_{A}=-i f_{A B C}\left(x_{s B} \partial_{s C}+\frac{1}{2} \theta_{\alpha B} \theta_{\alpha C}\right) .
\end{gathered}
$$

Here $\gamma^{s}$ are $16 \times 16$ dimensional, real matrices s.t. $\left\{\gamma^{s}, \gamma^{t}\right\}=2 \delta^{s t} \mathbf{1}_{16 \times 16}, \theta_{\alpha A}$ are Grassmann numbers s.t. $\left\{\theta_{\alpha A}, \theta_{\beta B}\right\}=\delta_{\alpha \beta} \delta_{A B}$, and $f_{A B C}$ are $S U(N)$ structure constants (real, antisymmetric). The physical (gauge invariant) Hilbert space consists of states $|\psi\rangle$ satisfying $J_{A}|\psi\rangle=0$.

An important question in matrix theory is related to the construction of the ground state. While there are serious indications that such state exists [5] and a number of results concerning its asymptotic form [6], the state has never been constructed analytically. In this talk we report on some progress towards this direction [7].

Using elliptic regularity (see e.g. [8]), one can write any solution to the equation $H \Psi=0$ in terms of a power series in the coordinates,

$$
\Psi(x)=\psi^{(0)}+x_{t A} \psi_{t A}^{(1)}+\frac{1}{2} x_{t A} x_{u B} \psi_{t A, u B}^{(2)}+\ldots
$$

with $\psi_{t_{1} A_{1} \ldots t_{k} A_{k}}^{(k)}$ depending entirely on the fermionic variables. Inserting this into the equation $\mathscr{Q}_{\hat{\beta}} \Psi=0$ we find a class of equations satisfied by $\psi_{t_{1} A_{1} \ldots t_{k} A_{k}}^{(k)}$, e.g.

$$
\begin{gathered}
\gamma_{\hat{\beta} \hat{\alpha}}^{t} \theta_{\hat{\alpha} A} \psi_{t A}^{(1)}=0, \quad \gamma_{\hat{\beta} \hat{\alpha}}^{t} \theta_{\hat{\alpha} A} \psi_{t A, u B}^{(2)}=0, \\
-i \gamma_{\hat{\beta} \hat{\alpha}}^{t} \theta_{\hat{\alpha} A} \psi_{t A, u B, v C}^{(3)}+\varepsilon_{A B C} \gamma_{\hat{\beta} \hat{\alpha}}^{u v} \theta_{\hat{\alpha} A} \psi^{(0)}=0,
\end{gathered}
$$

etc. for all $\hat{\beta}$. Solving the above equations would be an important result in understanding the behavior of the ground state around the origin. In the following part of this talk we will concentrate on the evaluation of $\psi^{(0)}$. 
The only constraints which $\psi^{(0)}$ satisfies follow from the requirement that $\psi^{(0)}$ has to be both the $S U(N)$ and the $\operatorname{Spin}(9)$ singlet. The corresponding equations are

$$
\underbrace{f_{A B C} \theta_{\alpha A} \theta_{\alpha B}}_{S U(N)} \psi^{(0)}=0, \quad \underbrace{\gamma_{\alpha \beta}^{s t} \theta_{\alpha A} \theta_{\alpha A}}_{S O(9)} \psi^{(0)}=0 .
$$

The existence and the uniqueness of such state for $S U(N=2)$ matrix theory was found earlier in [9] using symbolic programme. Here we present a group-theoretic construction of that result.

Out of the 16 real Majorana fermions one can build 8 creation and 8 annihilation fermionic operators and correspondingly construct the whole 256-dimensional fermionic Fock space $\mathscr{H}_{256}$. The state $\psi^{(0)}$ lies in the tensor product of three such spaces $\mathscr{H}_{256} \otimes \mathscr{H}_{256} \otimes \mathscr{H}_{256}$ each one corresponding to one color $A=1,2,3$. Under $\operatorname{Spin}(9)$ the representation 256 splits into $\mathbf{4 4}+\mathbf{8 4}+\mathbf{1 2 8}$. Let us denote by $|s t\rangle,|s t u\rangle$ and $|s \alpha\rangle$ vectors which span the $\mathbf{4 4 , 8 4}$ and 128 respectively (for their explicit construction see the Appendix in [7]). Clearly, any product of a type

$$
|s u\rangle_{1}|t u\rangle_{2}|s t\rangle_{3}, \quad|s \alpha\rangle_{1}|t \alpha\rangle_{2}|s t\rangle_{3},|s u v\rangle_{1}|t u v\rangle_{2}|s t\rangle_{3}
$$

(i.e. with no free indices) is a $\operatorname{Spin}(9)$ singlet and hence $\psi^{(0)}$ could be some linear combination of the above examples. However it turns out that the additional $S U(2)$ invariance picks up a unique combination, namely

$$
\left.\psi^{(0)} \propto \||||1\rangle+\frac{13}{36}|\|| 1\right\rangle
$$

where

$$
\begin{gathered}
|||1\rangle:=|s u\rangle_{1}|t u\rangle_{2}|s t\rangle_{3} \\
\left.\|_{844}|| 1\right\rangle:=|s u v\rangle_{1}|t u v\rangle_{2}|s t\rangle_{3}+|t u v\rangle_{1}|s t\rangle_{2}|s u v\rangle_{3}+|s t\rangle_{1}|s u v\rangle_{2}|t u v\rangle_{3}
\end{gathered}
$$

\section{References}

[1] P. A. Dirac, An Extensible Model Of The Electron, Proc. Roy. Soc. Lond. A268:57-67, 1962.

[2] J. Goldstone, unpublished,

J. Hoppe, Quantum Theory of a Massless Relativistic Surface and a two dimensional bound state problem, PhD Thesis MIT, (1982), (scanned version available at http://www.aei-potsdam.mpg.de/hoppe).

[3] D. de Wit, J. Hoppe, H. Nicolai, On the quantum mechanics of supermembranes, Nucl. Phys. B305 [FS23] (1988) 545-581.

[4] T. Banks, W. Fischler, S. Shenker and L. Susskind, M Theory As A Matrix Model: A Conjecture, Phys. Rev. D55 (1997) 6189, arXiv: hep-th/9610043.

[5] P. Yi, Witten Index and Threshold Bound States of D-Branes, Nucl. Phys. B505 (1997) 307-318, arXiv: hep-th/9704098,

S. Sethi, M. Stern, D-Brane Bound States Redux, Commun. Math. Phys. 194 (1998) 675-705, arXiv:hep-th/9705046,

M. B. Green, M. Gutperle, D-particle bound states and the D-instanton measure, JHEP 9801 (1998) 005, arXiv:hep-th/9711107, 
G. Moore, N. Nekrasov, S. Shatashvili, D-particle bound states and generalized instantons, Commun. Math. Phys. 209 (2000) 77-95, arXiv: hep-th/9803265,

V. G. Kac, A. V. Smilga, Normalized Vacuum States in $N=4$ Supersymmetric Yang-Mills Quantum Mechanics with Any Gauge Group, Nucl. Phys. B571 (2000) 515-554, arXiv : hep-th/9908096.

[6] J. Fröhlich, J. Hoppe, On Zero-Mass Ground States in Super-Membrane Matrix Models, arXiv:hep-th/9701119,

J. Hoppe, On the Construction of Zero Energy States in Supersymmetric Matrix Models, arXiv:hep-th/9709132,

M. B. Halpern, C. Schwartz, Asymptotic Search for Ground States of SU(2) Matrix Theory, Int. J. Mod. Phys. A13 (1998) 4367-4408, arXiv : hep-th/9712133,

A. Konechny, On Asymptotic Hamiltonian for SU(N) Matrix Theory, JHEP 9810 (1998) 018, arXiv:hep-th/9805046,

M. Bordemann, J. Hoppe, R. Suter, Zero Energy States for SU(N): A Simple Exercise in Group Theory ?, arXiv:hep-th/9909191,

J. Hoppe, Asymptotic Zero Energy States for $S U(N$ greater or equal 3), arXiv : hep-th/9912163, J. Fröhlich, G. M. Graf, D. Hasler, J. Hoppe, S.-T. Yau, Asymptotic form of zero energy wave functions in supersymmetric matrix models, Nucl. Phys. B567 (2000) 231-248, arXiv: hep-th/9904182,

J. Hoppe, J. Plefka, The Asymptotic Groundstate of SU(3) Matrix Theory, arXiv:hep-th/0002107,

D. Hasler, J. Hoppe, Asymptotic Factorisation of the Ground-State for SU(N)-invariant Supersymmetric Matrix-Models, arXiv : hep-th/0206043,

V. Bach, J. Hoppe, D. Lundholm, Dynamical Symmetries in Supersymmetric Matrix Models, arXiv:hep-th/07060355,

J. Hoppe, D. Lundholm, On the Construction of Zero Energy States in Supersymmetric Matrix Models IV, arXiv:0706.0353.

[7] J. Hoppe, D. Lundholm, M. Trzetrzelewski, Construction of the Zero-Energy State of SU(2)-Matrix Theory: Near the Origin, Nucl. Phys. B817:155-166, 2009 arXiv: 0809.5270.

[8] F. E. Browder, Strongly elliptic systems of differential equations, Annals of Mathematics Studies 33 (1954), 15-51.

[9] J. Wosiek, On the SO(9) structure of supersymmetric Yang-Mills quantum mechanics Phys. Lett. B619 (2005) 171-176, arXiv: hep-th/0503236. 